UT-Komaba/03-18

hep-th/0311206

\title{
Commuting Flows and Conservation Laws for Noncommutative Lax Hierarchies
}

\author{
Masashi Hamanaka \\ Institute of Physics, University of Tokyo, Komaba, \\ Meguro-ku, Tokyo 153-8902, Japan ${ }^{1}$
}

\begin{abstract}
We discuss commuting flows and conservation laws for Lax hierarchies on noncommutative spaces in the framework of the Sato theory. On commutative spaces, the Sato theory has revealed essential aspects of the integrability for wide class of soliton equations which are derived from the Lax hierarchies in terms of pseudo-differential operators. Noncommutative extension of the Sato theory has been already studied by the author and Kouichi Toda, and the existence of various noncommutative Lax hierarchies are guaranteed. In this paper, we present conservation laws for the noncommutative Lax hierarchies with both space-space and space-time noncommutativities and prove the existence of infinite number of conserved densities. We also give the explicit representations of them in terms of Lax operators. Our results include noncommutative versions of $\mathrm{KP}, \mathrm{KdV}$, Boussinesq, coupled KdV, Sawada-Kotera, modified KdV equations and so on.
\end{abstract}

\footnotetext{
${ }^{1}$ Present address: Graduate School of Mathematics, Nagoya University, Chikusa-ku, Nagoya, 4648602, JAPAN (E-mail: hamanaka@math.nagoya-u.ac.jp)
} 


\section{Introduction}

Non-Commutative (NC) extension of field theories has been studied intensively for the last several years [1]. NC gauge theories are equivalent to ordinary gauge theories in the presence of background magnetic fields and succeeded in revealing various aspects of them [2]. NC solitons especially play important roles in the study of D-brane dynamics, such as the confirmation of Sen's conjecture on tachyon condensation [3]. One of the distinguished features of NC theories is resolution of singularities. This gives rise to various new physical objects such as $U(1)$ instantons and makes it possible to analyze singular configurations as usual.

$\mathrm{NC}$ extension of integrable equations such as the Korteweg-de Vries (KdV) equation 4] is also one of the hot topics [5]-37]. These equations imply no gauge field and NC extension of them perhaps might have no physical picture or no good property on integrabilities. To make matters worse, the NC extension of $(1+1)$-dimensional equations introduces infinite number of time derivatives, which makes it hard to discuss or define the integrability. However, some of them actually possess integrable properties, such as the existence of infinite number of conserved quantities [7, 8, 9, 20] and the linearizability [30, 31] which are widely accepted as definition of completely integrability of equations. Furthermore, a few of them can be derived from NC (anti-)self-dual Yang-Mills (YM) equations by suitable reductions [14, 30, 33. This fact may give some physical meanings and good properties to the lower-dimensional NC field equations and makes us expect that the Ward conjecture 38] still holds on NC spaces [27]. So far, however, those equations have been examined one by one. Now it is very natural to discuss their integrabilities in more general framework.

The author and Kouichi Toda have studied systematic NC extension of integrable systems [27, 30, 36]. In the previous paper [36], we have obtained wide class of NC Lax hierarchies which include various $\mathrm{NC}$ versions of soliton equations in the framework of the Sato theory 39]. On commutative spaces, the Sato theory is known to be one of the most beautiful theories of solitons and reveals essential aspects of the integrability, such as, the construction of exact multi-soliton solutions, the structure of the solution space, the existence of infinite conserved quantities, and the hidden symmetry of them. In the Sato theory, the soliton equations are described by Lax hierarchies in terms of pseudo-differential operators. 
In the present paper, we prove the existence of infinite conserved quantities for Lax hierarchies on NC spaces in the framework of the Sato theory. We show the conservation laws for them and give the explicit representations with both space-space and space-time noncommutativities. This suggests that the NC soliton equations are also completely integrable and infinite-dimensional symmetries would be hidden. Our results include wide class of NC soliton equations, such as, NC versions of Kadomtsev-Petviashvili (KP) 40], KdV, Boussinesq 41], coupled KdV [42, Sawada-Kotera [4], modified KdV (mKdV) equations and so on.

\section{Comments on Noncommutative Field Theories}

NC spaces are defined by noncommutativity of the coordinates:

$$
\left[x^{i}, x^{j}\right]=i \theta^{i j}
$$

where $\theta^{i j}$ are real constants and called the $N C$ parameters.

$\mathrm{NC}$ field theories are obtained from given commutative field theories by exchange of ordinary products in the commutative field theories for star-products. The star-product is defined for ordinary fields on commutative spaces. On Euclidean spaces, it is explicitly given by

$$
\begin{aligned}
f(x) \star g(x) & :=f(x) \exp \left(\frac{i}{2} \overleftarrow{\partial}_{i} \theta^{i j} \vec{\partial}_{j}\right) g(x) \\
& =f(x) g(x)+\frac{i}{2} \theta^{i j} \partial_{i} f(x) \partial_{j} g(x)+\mathcal{O}\left(\theta^{2}\right)
\end{aligned}
$$

where $\partial_{i}:=\partial / \partial x^{i}$. This explicit representation is known as the Moyal product [4].

The star-product possesses associativity: $f \star(g \star h)=(f \star g) \star h$, and returns back to the ordinary product in the commutative limit: $\theta^{i j} \rightarrow 0$. The modification of the product makes the ordinary spatial coordinates "noncommutative," that is, $\left[x^{i}, x^{j}\right]_{\star}:=$ $x^{i} \star x^{j}-x^{j} \star x^{i}=i \theta^{i j}$.

We note that the fields themselves take c-numbers values and the differentiation and the integration for them are well-defined as usual. NC field theories should be interpreted as deformed theories from commutative ones. One of nontrivial points in the NC extension is order of nonlinear terms. The difference between commutative equations and the NC equations arise as commutators of fields which sometimes become serious obstructions. 
Here we point out a special property of the $\mathrm{NC}$ commutators of fields. It is convenient to introduce the following symbol

$$
P:=\frac{1}{2} \overleftarrow{\partial}_{i} \theta^{i j} \vec{\partial}_{j}
$$

and the Strachan product 45]

$$
f(x) \diamond g(x):=f(x)\left(\sum_{s=0}^{\infty} \frac{(-1)^{s}}{(2 s+1) !} P^{2 s}\right) g(x) .
$$

A commutator of fields is straightforwardly calculated as follows

$$
\begin{aligned}
{[f(x), g(x)]_{\star} } & =f(x)\left(e^{i P}-e^{-i P}\right) g(x)=: 2 i f(x)(\sin P) g(x) \\
& =-\theta^{i j} \partial_{i} f(x) \diamond \partial_{j} g(x) \\
& =-\theta^{i j} \partial_{i}\left(f(x) \diamond \partial_{j} g(x)\right) .
\end{aligned}
$$

In the second line, we use the fact that $\sin P$ is the composite of $P$ and " $P^{-1} \sin P$ " and the Strachan product " $\diamond$ " corresponds to the latter. This derivation was first presented by Dimakis and Müller-Hoissen in order to generate infinite number of conserved densities of the NC non-linear Schrödinger (NLS) equation [7, the NC KdV equation [8] and the NC extended matrix-NLS equation [9]. Here more widely, we would like to stress that commutators of fields on NC spaces always appear as total derivatives in the NC directions. This will be crucial in derivation of conservation laws in Sec. 5.

As a consequence, we can prove

$$
\int d^{D} x f(x) \star g(x)=\int d^{D} x f(x) g(x)
$$

where the integration is taken in all $\mathrm{NC}$ directions.

\section{Noncommutative Lax Hierarchies in Sato's Frame- work}

In this section, we derive various NC Lax equations in terms of pseudo-differential operators which include negative powers of differential operators. We note that the present discussion in this section can be applied to more general cases where the products are not necessarily the star-products but noncommutative associative products with differentiations, which has been already discussed in e.g. [46]. However, we believe that some explicit examples here are new equations and would be useful for further studies. 
An $N$-th order (monic) pseudo-differential operator $A$ is represented as follows

$$
A=\partial_{x}^{N}+a_{N-1} \partial_{x}^{N-1}+\cdots+a_{0}+a_{-1} \partial_{x}^{-1}+a_{-2} \partial_{x}^{-2}+\cdots .
$$

Here we introduce useful symbols:

$$
\begin{aligned}
A_{\geq r} & :=\partial_{x}^{N}+a_{N-1} \partial_{x}^{N-1}+\cdots+a_{r} \partial_{x}^{r}, \\
A_{\leq r} & :=A-A_{\geq r+1}=a_{r} \partial_{x}^{r}+a_{r-1} \partial_{x}^{r-1}+\cdots, \\
\operatorname{res}_{r} A & :=a_{r} .
\end{aligned}
$$

The symbol $\operatorname{res}_{-1} A$ is especially called the residue of $A$.

The action of a differential operator $\partial_{x}^{n}$ on a multiplicity operator $f$ is formally defined as the following generalized Leibniz rule:

$$
\partial_{x}^{n} \cdot f:=\sum_{i \geq 0}\left(\begin{array}{c}
n \\
i
\end{array}\right)\left(\partial_{x}^{i} f\right) \partial^{n-i}
$$

where the binomial coefficient is given by

$$
\left(\begin{array}{c}
n \\
i
\end{array}\right):=\frac{n(n-1) \cdots(n-i+1)}{i(i-1) \cdots 1} .
$$

We note that the definition of the binomial coefficient (3.6) is applicable to the case for negative $n$, which just define the action of negative power of differential operators. The examples are,

$$
\begin{aligned}
& \partial_{x}^{-1} \cdot f=f \partial_{x}^{-1}-f^{\prime} \partial_{x}^{-2}+f^{\prime \prime} \partial_{x}^{-3}-\cdots \\
& \partial_{x}^{-2} \cdot f=f \partial_{x}^{-2}-2 f^{\prime} \partial_{x}^{-3}+3 f^{\prime \prime} \partial_{x}^{-4}-\cdots \\
& \partial_{x}^{-3} \cdot f=f \partial_{x}^{-3}-3 f^{\prime} \partial_{x}^{-4}+6 f^{\prime \prime} \partial_{x}^{-5}-\cdots
\end{aligned}
$$

where $f^{\prime}:=\partial f / \partial x, f^{\prime \prime}:=\partial^{2} f / \partial x^{2}$ and so on, and $\partial_{x}^{-1}$ in the RHS acts as an integration operator $\int^{x} d x$.

The composition of pseudo-differential operators is also well-defined and the total set of pseudo-differential operators forms an operator algebra. For more on pseudo-differential operators and the Sato theory, see e.g. [47]-[49].

Let us introduce a Lax operator as the following first-order pseudo-differential operator:

$$
L=\partial_{x}+u_{1}+u_{2} \partial_{x}^{-1}+u_{3} \partial_{x}^{-2}+u_{4} \partial_{x}^{-3}+\cdots,
$$


where the coefficients $u_{k}(k=1,2, \ldots)$ are functions of infinite variables $\left(x^{1}, x^{2}, \ldots\right)$ with $x^{1} \equiv x:$

$$
u_{k}=u_{k}\left(x^{1}, x^{2}, \ldots\right)
$$

The noncommutativity is arbitrarily introduced for the variables $\left(x^{1}, x^{2}, \ldots\right)$ as Eq. (2.1) here.

The Lax hierarchy is defined in Sato's framework as

$$
\partial_{m} L=\left[B_{m}, L\right]_{\star}, \quad m=1,2, \ldots
$$

where the action of $\partial_{m}$ on the pseudo-differential operator $L$ should be interpreted to be coefficient-wise, that is, $\partial_{m} L:=\left[\partial_{m}, L\right]$ or $\partial_{m} \partial_{x}^{k}=0$. The operator $B_{m}$ is given by

$$
B_{m}:=(\underbrace{L \star \cdots \star L}_{m \text { times }})_{\geq r}=:\left(L^{m}\right)_{\geq r}
$$

where $r$ is 0 for $u_{1}=0$ and 1 for $u_{1} \neq 0$ as commutative cases [50, 51]. The Lax hierarchy gives rise to a set of infinite differential equations with respect to infinite kind of fields from the coefficients in Eq. (3.10) for a fixed $m$. Hence it contains huge amount of differential (evolution) equations for all $m$. The LHS of Eq. (3.10) becomes $\partial_{m} u_{k}$ which shows a flow in the $x^{m}$ direction.

If we put the constraint $L^{l}=B_{l}$ on the Lax hierarchy (3.10), we get infinite set of NC (reduced) Lax hierarchies. We can easily show

$$
\frac{\partial u_{k}}{\partial x^{N l}}=0
$$

for all $N, k$ because

$$
\frac{d L^{l}}{d x^{N l}}=\left[B_{N l}, L^{l}\right]_{\star}=\left[\left(L^{l}\right)^{N}, L^{l}\right]_{\star}=0
$$

which implies Eq. (3.12). The reduced NC hierarchy is called the l-reduction of the NC KP hierarchy. This time, the constraint $L^{l}=B_{l}$ gives simple relationships which make it possible to represent infinite kind of fields $u_{l-r+1}, u_{l-r+2}, u_{l-r+3}, \ldots$ in terms of $(l-1)$ kind of fields $u_{2-r}, u_{3-r}, \ldots, u_{l-r}$. (cf. Appendix A.)

From now on, let us see that those equations in the Lax hierarchy contain various soliton equations with some constraints. We discuss it separately in the following two 
cases: $u_{1}=0(r=0)$ case and $u_{1} \neq 0(r=1)$ case. Some of them are already discussed in [36]. For commutative discussions, see also [52].

For $u_{1}=0(r=0)$

In this case, the Lax hierarchy (3.10) is just the $N C$ KP hierarchy which includes the NC KP equation [16, 46]. Let us see it explicitly.

- NC KP hierarchy

The coefficients of each powers of (pseudo-)differential operators in the Lax hierarchy (3.10) yield a series of infinite NC "evolution equations," that is, for $m=1$

$$
\left.\partial_{x}^{1-k}\right) \quad \partial_{1} u_{k}=u_{k}^{\prime}, \quad k=2,3, \ldots \quad \Rightarrow \quad x^{1} \equiv x
$$

for $m=2$

$$
\begin{array}{ll}
\left.\partial_{x}^{-1}\right) & \partial_{2} u_{2}=u_{2}^{\prime \prime}+2 u_{3}^{\prime}, \\
\left.\partial_{x}^{-2}\right) & \partial_{2} u_{3}=u_{3}^{\prime \prime}+2 u_{4}^{\prime}+2 u_{2} \star u_{2}^{\prime}+2\left[u_{2}, u_{3}\right]_{\star}, \\
\left.\partial_{x}^{-3}\right) & \partial_{2} u_{4}=u_{4}^{\prime \prime}+2 u_{5}^{\prime}+4 u_{3} \star u_{2}^{\prime}-2 u_{2} \star u_{2}^{\prime \prime}+2\left[u_{2}, u_{4}\right]_{\star}, \\
\left.\partial_{x}^{-4}\right) & \partial_{2} u_{5}=\cdots,
\end{array}
$$

and for $m=3$

$$
\begin{aligned}
& \left.\partial_{x}^{-1}\right) \quad \partial_{3} u_{2}=u_{2}^{\prime \prime \prime}+3 u_{3}^{\prime \prime}+3 u_{4}^{\prime}+3 u_{2}^{\prime} \star u_{2}+3 u_{2} \star u_{2}^{\prime}, \\
& \left.\partial_{x}^{-2}\right) \quad \partial_{3} u_{3}=u_{3}^{\prime \prime \prime}+3 u_{4}^{\prime \prime}+3 u_{5}^{\prime}+6 u_{2} \star u_{3}^{\prime}+3 u_{2}^{\prime} \star u_{3}+3 u_{3} \star u_{2}^{\prime}+3\left[u_{2}, u_{4}\right]_{\star} \text {, } \\
& \left.\partial_{x}^{-3}\right) \quad \partial_{3} u_{4}=u_{4}^{\prime \prime \prime}+3 u_{5}^{\prime \prime}+3 u_{6}^{\prime}+3 u_{2}^{\prime} \star u_{4}+3 u_{2} \star u_{4}^{\prime}+6 u_{4} \star u_{2}^{\prime} \\
& -3 u_{2} \star u_{3}^{\prime \prime}-3 u_{3} \star u_{2}^{\prime \prime}+6 u_{3} \star u_{3}^{\prime}+3\left[u_{2}, u_{5}\right]_{\star}+3\left[u_{3}, u_{4}\right]_{\star} \text {, } \\
& \left.\partial_{x}^{-4}\right) \quad \partial_{3} u_{5}=\cdots
\end{aligned}
$$

These just imply the (2+1)-dimensional NC KP equation [16, 46] with $2 u_{2} \equiv u, x^{2} \equiv$ $y, x^{3} \equiv t:$

$$
\frac{\partial u}{\partial t}=\frac{1}{4} \frac{\partial^{3} u}{\partial x^{3}}+\frac{3}{4} \frac{\partial(u \star u)}{\partial x}+\frac{3}{4} \int^{x} d x^{\prime} \frac{\partial^{2} u\left(x^{\prime}\right)}{\partial y^{2}}-\frac{3}{4}\left[u, \int^{x} d x^{\prime} \frac{\partial u\left(x^{\prime}\right)}{\partial y}\right]_{\star} .
$$

Important point is that infinite kind of fields $u_{3}, u_{4}, u_{5}, \ldots$ are represented in terms of one kind of field $2 u_{2} \equiv u$ as is seen in Eq. (3.15). This guarantees the existence of NC KP hierarchy which implies the existence of reductions of the NC KP hierarchy. The order of nonlinear terms are determined in this way. 
- NC KdV Hierarchy (2-reduction of the NC KP hierarchy)

Taking the constraint $L^{2}=B_{2}=: \partial_{x}^{2}+u$ for the NC KP hierarchy, we get the NC $\mathrm{KdV}$ hierarchy. This time, the following NC Lax hierarchy

$$
\frac{\partial u}{\partial x^{m}}=\left[B_{m}, L^{2}\right]_{\star}
$$

include neither positive nor negative power of (pseudo-)differential operators for the same reason as commutative case (See e.g. [53].) and gives rise to the $m$-th $\mathrm{KdV}$ equation for each $m$. For example, the NC KdV hierarchy (3.18) becomes the $(1+1)$-dimensional NC KdV equation [8] for $m=3$ with $x^{3} \equiv t$

$$
\dot{u}=\frac{1}{4} u^{\prime \prime \prime}+\frac{3}{4}(u \star u)^{\prime}
$$

and the $(1+1)$-dimensional 5 -th NC KdV equation $\left[23\right.$ for $m=5$ with $x^{5} \equiv t$

$$
\dot{u}=\frac{1}{16} u^{\prime \prime \prime \prime \prime \prime}+\frac{5}{16}\left(u \star u^{\prime \prime \prime}+u^{\prime \prime \prime} \star u\right)+\frac{5}{8}\left(u^{\prime} \star u^{\prime}+u \star u \star u\right)^{\prime},
$$

where $\dot{u}:=\partial u / \partial t$.

- NC Boussinesq Hierarchy (3-reduction of the NC KP hierarchy)

The 3-reduction $L^{3}=B_{3}$ yields the NC Boussinesq hierarchy which includes the $(1+1)$-dimensional NC Boussinesq equation [23] with $t \equiv x^{2}$ :

$$
\ddot{u}=\frac{1}{3} u^{\prime \prime \prime \prime}+(u \star u)^{\prime \prime}+\left(\left[u, \partial_{x}^{-1} \dot{u}\right]_{\star}\right)^{\prime},
$$

where $\ddot{u}:=\partial^{2} u / \partial t^{2}$ and $\partial_{x}^{-1}=\int^{x} d x$.

- NC coupled KdV Hierarchy (4-reduction of the NC KP hierarchy)

The hierarchy includes the $(1+1)$-dimensional NC coupled $\mathrm{KdV}$ equation $t \equiv x^{3}$ :

$$
\dot{u}=\frac{1}{4} u^{\prime \prime \prime}+\frac{3}{4}(u \star u)^{\prime}+\frac{3}{4}\left(\omega-\phi^{2}\right)^{\prime}-\frac{3}{4}\left[u, \phi^{\prime}\right]_{\star},
$$

and other two equations with respect to three kind of fields $u, \omega$, and $\phi$, which are determined by Eqs. (3.15) and (3.16). The $x^{2}$-dependence of the fields is absorbed by the fields $\omega, \phi$.

In this way, we can generate infinite set of the $l$-reduced $\mathrm{NC}$ hierarchies. If we take other set-up, we can get many other hierarchies. 
- NC Sawada-Kotera hierarchy (3-reduction of the NC BKP hierarchy)

The NC version of BKP hierarchy [54] is obtained from the NC KP hierarchy by the constraint that the constant terms of $B_{m}$ for $m=1,3,5, \ldots$ should vanish. The 3-reduction of the NC BKP hierarchy includes the $(1+1)$-dimensional NC Sawada-Kotera equation with $t \equiv x^{5}, u \equiv 3 u_{2}$ :

$$
\dot{u}+\frac{1}{9} u^{\prime \prime \prime \prime \prime \prime}+\frac{5}{9} u^{\prime \prime \prime} \star u+\frac{5}{9} u^{\prime \prime} \star u^{\prime}+\frac{5}{9} u \star u^{\prime} \star u=0
$$

which is new.

For $u_{1} \neq 0(r=1)$

On commutative spaces, this situation generates modified KP (mKP) hierarchy and its reductions. On NC spaces, however, the existence of them is not always guaranteed. For the NC KP hierarchy, infinite kind of fields are described by one kind of from the $x^{2}$-flow equations (3.15). However this time the flow equation becomes

$$
\begin{aligned}
\left.\partial_{x}^{0}\right) & \partial_{2} u_{1}=u_{1}^{\prime \prime}+2 u_{2}^{\prime}+2 u_{1} \star u_{1}^{\prime}+2\left[u_{1}, u_{2}\right]_{\star}, \\
\left.\partial_{x}^{-1}\right) & \partial_{2} u_{2}=u_{2}^{\prime \prime}+2 u_{3}^{\prime}+2 u_{1} \star u_{2}^{\prime}+2\left[u_{1}, u_{3}\right]_{\star}, \\
\left.\partial_{x}^{-2}\right) & \partial_{2} u_{3}=\cdots
\end{aligned}
$$

Hence due to the commutator $\left[u_{1}, u_{k}\right]$, it is very hard to represent the field $u_{k}$ in terms of $u_{1}, u_{2}, \ldots, u_{k-1}$. The same is true of other flows. That is why the existence of NC modified KP hierarchy is nontrivial.

Some reduced hierarchies are obtained from constraint conditions.

- NC mKdV Hierarchy (2-reduction of the "NC mKP hierarchy")

This time, the 2-reduction constraint $L^{2}=B_{2}$ makes it possible to represent infinite kind of fields $u_{2}, u_{3}, \ldots$ in terms of one kind of field $2 u_{1} \equiv v$. The $\mathrm{NC} \mathrm{mKdV}$ hierarchy includes the $(1+1)$-dimensional $\mathrm{NC}$ mKdV equation for $m=3$ with $t_{3} \equiv t:$

$$
\dot{v}=\frac{1}{4} v^{\prime \prime \prime}-\frac{3}{8} v \star v^{\prime} \star v+\frac{3}{8}\left[v, v^{\prime \prime}\right]_{\star} .
$$

- NC Burgers Hierarchy 30. 
This is obtained by an irregular reduction. Putting the constraint $L_{\leq-1}=0$ or $L=: \partial_{x}+v$, the Lax hierarchy (3.10) yields the NC Burgers hierarchy which includes neither positive nor negative power of differential operator. For $m=2$, the hierarchy becomes the $(1+1)$-dimensional NC Burgers equation with $t \equiv x^{2}$ :

$$
\dot{v}=\left[B_{2}, L\right]_{\star}=\left[\partial_{x}^{2}+2 v \partial_{x}, \partial_{x}+v\right]_{\star}=v^{\prime \prime}+2 v \star v^{\prime} .
$$

The NC Burgers equation is linearizable and easily solved via NC Cole-Hopf transformation [30, 31]. In the linearization, the order of the nonlinear term play crucial roles. This order is automatically realized from Sato's framework.

The present discussion is applicable to the matrix Sato theory where the fields $u_{k}(k=$ $1,2, \ldots)$ are $N \times N$ matrices. For $N=2$, the Lax hierarchy includes the Ablowitz-KaupNewell-Segur (AKNS) system [55], the Davey-Stewarson equation, the NLS equation and so on. (For commutative discussions, see e.g. 48].)

$\mathrm{NC}$ version [23] of the Bogoyavlenskii-Calogero-Schiff (BCS) equation [56] is also derived from this framework because the Sato theory works well on the commutative BCS equation.

\section{Commuting Flows for NC Lax Hierarchies}

First let us show all flows are commuting:

$$
\partial_{m} \partial_{n} u_{k}=\partial_{n} \partial_{m} u_{k}
$$

for any $m, n, k$. The derivation in this section is straightforward as commutative case [57, 53] and already discussed in more general situation where the products are noncommutative associative products with differentiations. (See e.g. [46, 58, 59].)

From NC Lax equation (3.10), we get

$$
\partial_{m} \partial_{n} L=\left[\partial_{m} B_{n}, L\right]_{\star}+\left[B_{n}, \partial_{m} L\right]_{\star}=\left[\partial_{m} B_{n}, L\right]_{\star}+\left[B_{n},\left[B_{m}, L\right]_{\star}\right]_{\star} .
$$

Hence

$$
\left[\partial_{m}, \partial_{n}\right] L=\left[F_{m n}, L\right]_{\star}
$$

where

$$
F_{m n}:=\partial_{m} B_{n}-\partial_{n} B_{m}-\left[B_{m}, B_{n}\right]_{\star}
$$


Now we show the "zero-curvature equation" $F_{m n}=0$. We note that

$$
\begin{aligned}
\partial_{m} B_{n} & =\partial_{m}\left(L^{n}\right)_{\geq r}=\left(\partial_{m} L^{n}\right)_{\geq r} \\
& =\left[B_{m}, L^{n}\right]_{\star \geq r}=-\left[B_{m}^{c}, L^{n}\right]_{\star \geq r} \\
& =-\left[B_{m}^{c}, B_{n}\right]_{\star \geq \geq r},
\end{aligned}
$$

where the operator $B_{m}^{c}$ is the compliment of $B_{m}$ and defined by

$$
B_{m}^{c}:=L^{m}-B_{m},
$$

and the suffix $r$ is equal to 0 for $u_{1}=0$ and 1 for $u_{1} \neq 0$. Therefore we get

$$
\begin{aligned}
F_{m n} & =-\left[B_{m}^{c}, B_{n}\right]_{\star \geq r}+\left[B_{n}^{c}, B_{m}\right]_{\star \geq r}-\left[B_{m}, B_{n}\right]_{\star} \\
& =-\left[B_{m}^{c}, L^{n}-B_{n}^{c}\right]_{\star \geq r}+\left[L^{n}-B_{n}, B_{m}\right]_{\star \geq r}-\left[B_{m}, B_{n}\right]_{\star \geq r} \\
& =\left[B_{m}^{c}, B_{n}^{c}\right]_{\star \geq r} \\
& =0
\end{aligned}
$$

which implies

$$
\partial_{m} \partial_{n} L=\partial_{n} \partial_{m} L
$$

Hence Eq. (4.1) is proved.

We note that the present discussion works well for arbitrary noncommutativity. Here we call the equation (4.7) the NC Zakharov-Shabat equation because it reduces to the usual Zakharov-Shabat equation in the commutative limit:

$$
\partial_{m} B_{n}-\partial_{n} B_{m}-\left[B_{m}, B_{n}\right]_{\star}=0 .
$$

Of course, we can get the conjugate of the NC Zakharov-Shabat equation in terms of $B_{n}^{c}$ :

$$
\partial_{m} B_{n}^{c}-\partial_{n} B_{m}^{c}+\left[B_{m}^{c}, B_{n}^{c}\right]_{\star}=0 .
$$

\section{Conservation Laws for NC Lax Hierarchies}

Here let us prove the conservation laws for NC Lax equations, which are the main results in the present paper, 
First we would like to comment on conservation laws of NC field equations [30]. The discussion is basically the same as commutative case because both the differentiation and the integration are the same as commutative ones in the Moyal representation.

Let us suppose the conservation law

$$
\frac{\partial \sigma\left(t, x^{i}\right)}{\partial t}=\partial_{i} J^{i}\left(t, x^{i}\right)
$$

where $\sigma\left(t, x^{i}\right)$ and $J^{i}\left(t, x^{i}\right)$ are called the conserved density and the associated flux, respectively. The conserved quantity is given by spatial integral of the conserved density:

$$
Q(t)=\int_{\text {space }} d^{D} x \sigma\left(t, x^{i}\right),
$$

where the integral $\int_{\text {space }} d x^{D}$ is taken for spatial coordinates. The proof is straightforward:

$$
\frac{d Q}{d t}=\frac{\partial}{\partial t} \int_{\text {space }} d^{D} x \sigma\left(t, x^{i}\right)=\int_{\text {space }} d^{D} x \partial_{i} J_{i}\left(t, x^{i}\right)=\int_{\text {infinity }} d S^{i} J_{i}\left(t, x^{i}\right)=0,
$$

unless the surface term of the integrand $J_{i}\left(t, x^{i}\right)$ vanishes. The convergence of the integral is also expected because the star-product naively reduces to the ordinary product at spatial infinity due to: $\partial_{i} \sim \mathcal{O}\left(r^{-1}\right)$ where $r:=|x|$.

For commutative field equations, the existence of infinite number of conserved quantities is expected to lead to infinite-dimensional hidden symmetry from Noether's theorem. For NC field equations, this would be also true and the existence of infinite number of conserved quantities would be special and meaningful, and suggest an infinite-dimensional hidden symmetry deformed from commutative one.

In order to discuss conservation laws for the NC Lax hierarchies, let us first calculate the differential of the residue of $L^{n}$ following G. Wilson's approach [57]:

$$
\partial_{m} \operatorname{res}_{-1} L^{n}=\operatorname{res}_{-1}\left(\partial_{m} L^{n}\right)=\operatorname{res}_{-1}\left[B_{m}, L^{n}\right]_{\star} .
$$

Here we note that

$$
\begin{aligned}
\operatorname{res}_{-1}\left[f \partial_{x}^{p}, g \partial_{x}^{q}\right]_{\star} & =\left(\begin{array}{c}
p \\
p+q+1
\end{array}\right)\left(f \star g^{(p+q+1)}-(-1)^{p+q+1} g \star f^{(p+q+1)}\right) \\
& =\left(\begin{array}{c}
p \\
p+q+1
\end{array}\right)\left\{\left(\sum_{k=0}^{p+q}(-1)^{k} f^{(k)} \star g^{(p+q-k)}\right)^{\prime}+(-1)^{p+q}\left[g, f^{(p+q+1)}\right]_{\star}\right\},
\end{aligned}
$$

where $f^{(N)}:=\partial^{N} f / \partial x^{N}$ Hence we can see that on NC spaces, there is an additional term as a commutator in Eq. (5.5) which vanishes in commutative limit. However as we saw 
in Sec. 2, commutators of fields can be represented as total derivatives, which is very important here.

Let us describe the explicit representations of the conservation laws. From the explicit forms of the Lax pair

$$
\begin{aligned}
L^{n} & =\partial_{x}^{n}+\sum_{l=1}^{\infty} a_{n-l} \partial_{x}^{n-l} \\
B_{m} & =\partial_{x}^{m}+\sum_{k=1}^{m} b_{m-k} \partial_{x}^{m-k}
\end{aligned}
$$

we can evaluate Eq. (15.4) as:

$$
\begin{aligned}
& \partial_{m} \operatorname{res}_{-1} L^{n}=\operatorname{res}_{-1}\left[\partial_{x}^{m}+\sum_{k=1}^{m} b_{m-k} \partial_{x}^{m-k}, \partial_{x}^{n}+\sum_{l=1}^{\infty} a_{n-l} \partial_{x}^{n-l}\right]_{\star} \\
& =\sum_{l=n+1}^{m+n}\left(\begin{array}{c}
m \\
l-n-1
\end{array}\right) a_{n-l}^{(m+n-l+1)}+\sum_{k=1}^{m} \sum_{l=n+1}^{n+1+m-k}\left(\begin{array}{c}
m-k \\
l-n-1
\end{array}\right) \\
& \times\left\{\left(\sum_{N=0}^{m+n-k-l}(-1)^{N} b_{m-k}^{(N)} \star a_{n-l}^{(m+n-k-l-N)}\right)^{\prime}+(-1)^{m+n-k-l}\left[a_{n-l}, b_{m-k}^{(m+n-k-l+1)}\right]_{\star}\right\} \\
& =\left\{\sum_{l=n+1}^{m+n}\left(\begin{array}{c}
m \\
l-n-1
\end{array}\right) a_{n-l}^{(m+n-l)}+\sum_{k=1}^{m} \sum_{l=n+1}^{m-k}\left(\begin{array}{c}
m-k \\
l-n-1
\end{array}\right) \sum_{N=0}^{m+1+}(-1)^{N} b_{m-k}^{(N)} \star a_{n-l}^{(m+n-k-l-N)}\right\}^{-k+l} \\
& -\sum_{k=1}^{m} \sum_{l=n+1}^{n+1+m-k}\left(\begin{array}{c}
m-k \\
l-n-1
\end{array}\right)(-1)^{m+n-k-l} \theta^{i j} \partial_{i}\left(a_{n-l} \diamond \partial_{j} b_{m-k}^{(m+n-k-l+1)}\right)
\end{aligned}
$$

This is the generalized conservation laws for the NC Lax hierarchies. The RHS contains derivatives in all NC directions. When we interpreted this as conservation laws, we have to specify what coordinates correspond to time and space and introduce the noncommutativities in the space-time directions only.

If we identify the coordinate $x^{m}$ with time $t$, we get the conserved density as follows:

$$
\sigma=\operatorname{res}_{-1} L^{n}+\theta^{i m} \sum_{k=0}^{m-1} \sum_{l=0}^{k}(-1)^{k-l}\left(\begin{array}{c}
k \\
l
\end{array}\right) \operatorname{res}_{-(l+1)} L^{n} \diamond \partial_{i} \partial_{x}^{k-l} \operatorname{res}_{k} L^{m},
$$

for $n=1,2, \ldots$, where the suffices $i$ must run in the space-time directions only. We can easily see that deformation terms appear in the second term of Eq. (5.7) in the case of space-time noncommutativity. On the other hand, in the case of space-space noncommutativity, the conserved density is given by the residue of $L^{n}$ as commutative case.

Let us show more explicit representations as follows. 
- In the case that the space-time coordinates are $(x, y, t) \equiv\left(x^{1}, x^{2}, x^{3}\right)$

The conserved density is given by

$$
\sigma=\operatorname{res}_{-1} L^{n}+\theta^{i m} \sum_{k=0}^{2} \sum_{l=0}^{k}(-1)^{k-l}\left(\begin{array}{c}
k \\
l
\end{array}\right) \text { res }{ }_{-(l+1)} L^{n} \diamond \partial_{i} \partial_{x}^{k-l} \operatorname{res}_{k} L^{3},
$$

more explicitly, for $u_{1}=0$ and $[t, x]=i \theta$, which includes the NC KP equation with space-time noncommutativity, the NC KdV equation and so on:

$$
\sigma=\operatorname{res}_{-1} L^{n}-3 \theta\left(\left(\operatorname{res}_{-1} L^{n}\right) \diamond u_{3}^{\prime}+\left(\operatorname{res}_{-2} L^{n}\right) \diamond u_{2}^{\prime}\right)
$$

and for $u_{1} \neq 0$ and $[t, x]=i \theta$, which includes the NC modified KdV equation and so on:

$$
\begin{aligned}
\sigma= & \operatorname{res}_{-1} L^{n} \\
& +3 \theta\left(\left(\operatorname{res}_{-1} L^{n}\right) \diamond\left(u_{2}+u_{1}^{2}\right)^{\prime \prime}-\left(\operatorname{res}_{-2} L^{n}\right) \diamond\left(u_{2}-u_{1}^{\prime}-u_{1}^{2}\right)^{\prime}-\left(\operatorname{res}_{-3} L^{n}\right) \diamond u_{1}^{\prime}\right) .
\end{aligned}
$$

- In the case that the space-time coordinates are $(x, t) \equiv\left(x^{1}, x^{2}\right)$ with $[t, x]=i \theta$ The conserved density is given by

$$
\sigma=\operatorname{res}_{-1} L^{n}-\theta \sum_{k=0}^{1} \sum_{l=0}^{k}(-1)^{k-l}\left(\begin{array}{c}
k \\
l
\end{array}\right) \operatorname{res}_{-(l+1)} L^{n} \diamond \partial_{i} \partial_{x}^{k-l} \operatorname{res}_{k} L^{2},
$$

more explicitly, for $u_{1}=0$, which includes the NC Boussinesq equation and so on:

$$
\sigma=\operatorname{res}_{-1} L^{n}+2 \theta\left(\operatorname{res}_{-1} L^{n}\right) \diamond u_{2}^{\prime}
$$

and for $u_{1} \neq 0$ :

$$
\sigma=\operatorname{res}_{-1} L^{n}+2 \theta\left(\left(\operatorname{res}_{-1} L^{n}\right) \diamond u_{1}^{\prime \prime}-\left(\operatorname{res}_{-2} L^{n}\right) \diamond u_{1}^{\prime}\right)
$$

We note that for space-space noncommutativity, conserved quantities (not densities) are all the same as commutative ones because of Eq. (2.6). This is consistent with the present results, of course. Furthermore, for $l$-reduced hierarchies, the conserved densities (5.7) become trivial for $n=N l(N=1,2, \ldots)$. The NC Burgers hierarchy is obtained by a "1-reduction" and contains no negative power of differential operators. Hence we cannot generate any conserved density for the NC Burgers equation in the present approach. This 
is considered to suggest that the NC Burgers equation is not a conservative system but a dispersive system as commutative case.

We have one comment on conserved densities for one-soliton configuration. One soliton solutions can always reduce to the commutative ones because $f(t-x) \star g(t-x)=f(t-$ $x) g(t-x)$ [8, 30]. Hence the conserved densities are not deformed in the NC extension.

The present discussion is applicable to the NC matrix Sato theory, including the NC AKNS system, the NC Davey-Stewarson equation, the NC NLS equation, and the NC BCS equation.

\section{Conclusion and Discussion}

In the present paper, we showed that the existence of infinite number of conserved densities for wide class of NC Lax hierarchies and obtained the explicit representations of them for both space-space and space-time noncommutativities. This suggests that NC soliton equations are completely integrable and infinite-dimensional symmetries would be hidden, which would be considered as some deformed affine Lie algebras.

In order to reveal what the hidden symmetry is, we have to first study NC extension of Hirota's bilinearization [60]. This could be realized as a simple generalization of the Cole-Hope transformation whose extension to NC spaces are already successful in [30, 31. Hirota's bilinearization leads to the theory of tau-functions which is essential in the discussion of the Lie algebraic structure of symmetry of the solution space [47, 54, 61, 62. After submission of the present manuscript to this journal, progress has been reported in e.g. 63, 64, 65].

Our results guarantee that NC extension of soliton theories would be actually fruitful and worth studying. There are many further directions, such as, the study of relation to $q$-deformations of integrable systems, $\mathrm{NC}$ extension of the $r$-matrix formalism [48, 66], the inverse scattering method and the Bäcklund transformation, and so on. NC extension of the Ward conjecture [38] (See also 67]) would be also very interesting [27. Some NC equations are actually derived from NC (anti-)self-dual YM equations by reduction [14, 30, 33] and embedded [15, 17, 68] in $N=2$ string theories 69]. This guarantees that NC soliton equations would have physical meanings and might be helpful to understand new aspects of the corresponding string theory. 


\section{Acknowledgements}

The author would like to thank H. Awata, H. Ishikawa, S. Kakei, M. Kato, I. Kishimoto, A. Nakamula, S. Odake, R. Sasaki, J. Shiraishi, K. Toda, T. Tsuchida, M. Wadati and S. Watamura for useful comments, and A. Dimakis and F. Müller-Hoissen for valuable remarks via e-mail correspondence. He is also grateful to organizers and audiences during the workshops YITP-W-03-07 on "QFT 2003" and YITP-W-04-03 on "QFT 2004" for hospitality and discussion. This work was supported by JSPS Research Fellowships for Young Scientists (\#0310363).

\section{A Miscellaneous Formulas}

We present explicit calculations of $L^{n}$ for $n=1,2,3,4,5$ up to some order of the pseudodifferential operator $\partial_{x}$. We can read reduction conditions, e.g. $L^{l}=B_{l}$, and the explicit representations of $\operatorname{res}_{r} L^{n}$ and $B_{m}$.

For $u_{1}=0(r=0)$ :

$$
\begin{aligned}
L= & \partial_{x}+u_{2} \partial_{x}^{-1}+u_{3} \partial_{x}^{-2}+u_{4} \partial_{x}^{-3}+u_{5} \partial_{x}^{-4}+u_{6} \partial_{x}^{-5}+\cdots, \\
L^{2}= & \partial_{x}^{2}+2 u_{2}+\left(2 u_{3}+u_{2}^{\prime}\right) \partial_{x}^{-1}+\left(2 u_{4}+u_{3}^{\prime}+u_{2} \star u_{2}\right) \partial_{x}^{-2} \\
& +\left(2 u_{5}+u_{4}^{\prime}+u_{2} \star u_{3}+u_{3} \star u_{2}-u_{2} \star u_{2}^{\prime}\right) \partial_{x}^{-3} \\
& +\left(2 u_{6}+u_{5}^{\prime}+u_{2} \star u_{4}+u_{4} \star u_{2}+u_{3} \star u_{3}-u_{2} \star u_{3}^{\prime}-2 u_{3} \star u_{2}^{\prime}+u_{2} \star u_{2}^{\prime \prime}\right) \partial_{x}^{-4}+\cdots, \\
L^{3}= & \partial_{x}^{3}+3 u_{2} \partial_{x}+3\left(u_{3}+u_{2}^{\prime}\right)+\left(3 u_{4}+3 u_{3}^{\prime}+u_{2}^{\prime \prime}+3 u_{2} \star u_{2}\right) \partial_{x}^{-1} \\
& +\left(3 u_{5}+3 u_{4}^{\prime}+u_{3}^{\prime \prime}+3 u_{2} \star u_{3}+3 u_{3} \star u_{2}+u_{2}^{\prime} \star u_{2}-u_{2} \star u_{2}^{\prime}\right) \partial_{x}^{-2} \\
& +\left(3 u_{6}+3 u_{5}^{\prime}+u_{4}^{\prime \prime}+3 u_{2} \star u_{4}+3 u_{4} \star u_{2}+3 u_{3} \star u_{3}+u_{2} \star u_{2} \star u_{2}\right. \\
& \left.+u_{2}^{\prime} \star u_{3}-u_{2} \star u_{3}^{\prime}+u_{3}^{\prime} \star u_{2}-4 u_{3} \star u_{2}^{\prime}-u_{2}^{\prime} \star u_{2}^{\prime}+u_{2} \star u_{2}^{\prime \prime}\right) \partial_{x}^{-3}+\cdots, \\
L^{4}= & \partial_{x}^{4}+4 u_{2} \partial_{x}^{2}+\left(4 u_{3}+6 u_{2}^{\prime}\right) \partial_{x}+\left(4 u_{4}+6 u_{3}^{\prime}+4 u_{2}^{\prime \prime}+6 u_{2} \star u_{2}\right) \\
& +\left(4 u_{5}+6 u_{4}^{\prime}+4 u_{3}^{\prime \prime}+u_{2}^{\prime \prime \prime}+6 u_{2} \star u_{3}+6 u_{3} \star u_{2}+4 u_{2}^{\prime} \star u_{2}+2 u_{2} \star u_{2}^{\prime}\right) \partial_{x}^{-1} \\
& +\left(4 u_{6}+6 u_{5}^{\prime}+4 u_{4}^{\prime \prime}+u_{3}^{\prime \prime \prime}+6 u_{2} \star u_{4}+6 u_{4} \star u_{2}+6 u_{3} \star u_{3}+4 u_{2} \star u_{2} \star u_{2}\right. \\
& \left.+4 u_{2}^{\prime} \star u_{3}+2 u_{2} \star u_{3}^{\prime}+4 u_{3}^{\prime} \star u_{2}-4 u_{3} \star u_{2}^{\prime}-u_{2}^{\prime} \star u_{2}^{\prime}+u_{2}^{\prime \prime} \star u_{2}+u_{2} \star u_{2}^{\prime \prime}\right) \partial_{x}^{-2}+\cdots, \\
L^{5}= & \partial_{x}^{5}+5 u_{2} \partial_{x}^{3}+5\left(u_{3}+2 u_{2}^{\prime}\right) \partial_{x}^{2}+5\left(u_{4}+2 u_{3}^{\prime}+2 u_{2}^{\prime \prime}+2 u_{2} \star u_{2}\right) \partial_{x} \\
& +5\left(u_{5}+2 u_{4}^{\prime}+2 u_{3}^{\prime \prime}+u_{2}^{\prime \prime \prime}+2 u_{2} \star u_{3}+2 u_{3} \star u_{2}+2 u_{2}^{\prime} \star u_{2}+2 u_{2} \star u_{2}^{\prime}\right)
\end{aligned}
$$




$$
\begin{aligned}
& +\left(5 u_{6}+10 u_{5}^{\prime}+10 u_{4}^{\prime \prime}+5 u_{3}^{\prime \prime \prime}+u_{2}^{\prime \prime \prime \prime}+10 u_{2} \star u_{4}+10 u_{4} \star u_{2}+10 u_{3} \star u_{3}+10 u_{2} \star u_{2} \star u_{2}\right. \\
& \left.+10 u_{2}^{\prime} \star u_{3}+10 u_{2} \star u_{3}^{\prime}+10 u_{3}^{\prime} \star u_{2}+5 u_{2}^{\prime} \star u_{2}^{\prime}+5 u_{2}^{\prime \prime} \star u_{2}+5 u_{2} \star u_{2}^{\prime \prime}\right) \partial_{x}^{-1}+\cdots .
\end{aligned}
$$

For $u_{1} \neq 0(r=1)$ :

$$
\begin{aligned}
& L=\partial_{x}+u_{1}+u_{2} \partial_{x}^{-1}+u_{3} \partial_{x}^{-2}+u_{4} \partial_{x}^{-3}+u_{5} \partial_{x}^{-4}+u_{6} \partial_{x}^{-5}+\cdots, \\
& L^{2}=\partial_{x}^{2}+2 u_{1} \partial_{x}+\left(2 u_{2}+u_{1}^{\prime}+u_{1}^{2}\right)+\left(2 u_{3}+u_{2}^{\prime}+u_{1} \star u_{2}+u_{2} \star u_{1}\right) \partial_{x}^{-1} \\
& +\left(2 u_{4}+u_{3}^{\prime}+u_{1} \star u_{3}+u_{3} \star u_{1}+u_{2} \star u_{2}-u_{2} \star u_{1}^{\prime}\right) \partial_{x}^{-2} \\
& +\left(2 u_{5}+u_{4}^{\prime}+u_{1} \star u_{4}+u_{4} \star u_{1}+u_{2} \star u_{3}+u_{3} \star u_{2}-2 u_{3} \star u_{1}^{\prime}-u_{2} \star u_{2}^{\prime}+u_{2} \star u_{1}^{\prime \prime}\right) \partial_{x}^{-3} \\
& +\cdots \text {, } \\
& L^{3}=\partial_{x}^{3}+3 u_{1} \partial_{x}^{2}+3\left(u_{2}+u_{1}^{\prime}+u_{1} \star u_{1}\right) \partial_{x} \\
& +\left(3 u_{3}+3 u_{2}^{\prime}+3 u_{1}^{\prime \prime}+3 u_{1} \star u_{2}+3 u_{2} \star u_{1}+u_{1}^{\prime} \star u_{1}+2 u_{1} \star u_{1}^{\prime}+u_{1} \star u_{1} \star u_{1}\right) \\
& +\left(3 u_{4}+3 u_{3}^{\prime}+u_{2}^{\prime \prime}+3 u_{1} \star u_{3}+3 u_{3} \star u_{1}+3 u_{2} \star u_{2}+u_{1}^{\prime} \star u_{2}+2 u_{1} \star u_{2}^{\prime}\right. \\
& \left.+u_{2}^{\prime} \star u_{1}-2 u_{2} \star u_{1}^{\prime}+u_{1} \star u_{1} \star u_{2}+u_{1} \star u_{2} \star u_{1}+u_{2} \star u_{1} \star u_{1}\right) \partial_{x}^{-1}+\cdots, \\
& L^{4}=\partial_{x}^{4}+4 u_{1} \partial_{x}^{3}+\left(4 u_{2}+6 u_{1}^{\prime}+6 u_{1} \star u_{1}\right) \partial_{x}^{2} \\
& +\left(4 u_{3}+6 u_{2}^{\prime}+4 u_{1}^{\prime \prime}+6 u_{1} \star u_{2}+6 u_{2} \star u_{1}+4 u_{1}^{\prime} \star u_{1}+8 u_{1} \star u_{1}^{\prime}+4 u_{1} \star u_{1} \star u_{1}\right) \partial_{x} \\
& +\left(4 u_{4}+6 u_{3}^{\prime}+4 u_{2}^{\prime \prime}+u_{1}^{\prime \prime \prime}+6 u_{1} \star u_{3}+6 u_{3} \star u_{1}+6 u_{2} \star u_{2}\right. \\
& +4 u_{1}^{\prime} \star u_{2}+6 u_{1} \star u_{2}^{\prime}+4 u_{2}^{\prime} \star u_{1}-2 u_{2} \star u_{1}^{\prime}+2 u_{1}^{\prime \prime} \star u_{1}+2 u_{1} \star u_{1}^{\prime \prime}+3 u_{1}^{\prime} \star u_{1}^{\prime} \\
& +4 u_{1} \star u_{1} \star u_{2}+4 u_{1} \star u_{2} \star u_{1}+4 u_{2} \star u_{1} \star u_{1}+u_{1}^{\prime} \star u_{1} \star u_{1}+2 u_{1} \star u_{1}^{\prime} \star u_{1} \\
& \left.+3 u_{1} \star u_{1} \star u_{1}^{\prime}+u_{1} \star u_{1} \star u_{1} \star u_{1}\right)+\cdots, \\
& L^{5}=\partial_{x}^{5}+5 u_{1} \partial_{x}^{4}+5\left(u_{2}+2 u_{1}^{\prime}+2 u_{1} \star u_{1}\right) \partial_{x}^{3} \\
& +5\left(u_{3}+2 u_{2}^{\prime}+2 u_{1}^{\prime \prime}+2 u_{1} \star u_{2}+2 u_{2} \star u_{1}+2 u_{1}^{\prime} \star u_{1}+4 u_{1} \star u_{1}^{\prime}+2 u_{1} \star u_{1} \star u_{1}\right) \partial_{x}^{2} \\
& +\left(5 u_{4}+10 u_{3}^{\prime}+10 u_{2}^{\prime \prime}+5 u_{1}^{\prime \prime \prime}+10 u_{1} \star u_{3}+10 u_{3} \star u_{1}+10 u_{2} \star u_{2}\right. \\
& +10 u_{1}^{\prime} \star u_{2}+20 u_{1} \star u_{2}^{\prime}+10 u_{2}^{\prime} \star u_{1}+4 u_{2} \star u_{1}^{\prime}+6 u_{1}^{\prime \prime} \star u_{1}+15 u_{1}^{\prime} \star u_{1}^{\prime}+11 u_{1} \star u_{1}^{\prime \prime} \\
& +10 u_{1} \star u_{1} \star u_{2}+10 u_{1} \star u_{2} \star u_{1}+10 u_{2} \star u_{1} \star u_{1} \\
& \left.+5 u_{1}^{\prime} \star u_{1} \star u_{1}+10 u_{1} \star u_{1}^{\prime} \star u_{1}+15 u_{1} \star u_{1} \star u_{1}^{\prime}+5 u_{1} \star u_{1} \star u_{1} \star u_{1}\right) \partial_{x}+\cdots .
\end{aligned}
$$

\section{References}

[1] M. R. Douglas and N. A. Nekrasov, Rev. Mod. Phys. 73 (2002) 977 hep-th/0106048;

R. J. Szabo, Phys. Rept. 378 (2003) 207 hep-th/0109162 and references therein. 
[2] K. Furuuchi, "Topological charge of U(1) instantons on noncommutative $\mathbf{R}^{4}$," hep-th/0010006 N. A. Nekrasov, "Trieste lectures on solitons in noncommutative gauge theories," hep-th/0011095 A. Konechny and A. Schwarz, Phys. Rept. 360 (2002) 353 hep-th/0107251; M. Hamanaka, "Noncommutative solitons and Dbranes," Ph. D thesis, hep-th/0303256; F. A. Schaposnik, "Noncommutative solitons and instantons," hep-th/0310202 and references therein.

[3] J. A. Harvey, "Komaba lectures on noncommutative solitons and D-branes," hep-th/0102076 and references therein.

[4] D. J. Korteweg and G. de Vries, Phil. Mag. 39 (1895) 422.

[5] T. Curtright, D. Fairlie and C. K. Zachos, Phys. Lett. B 405 (1997) 37 hep-th/9704037;; C. K. Zachos, D. Fairlie and T. Curtright, "Matrix membranes and integrability," hep-th/9709042.

[6] A. Dimakis and F. Müller-Hoissen, Int. J. Mod. Phys. B 14 (2000) 2455 hep-th/0006005; Lett. Math. Phys. 54 (2000) 123 hep-th/0007160; J. Phys. A 34 (2001) 9163 nlin.si/0104071.

[7] A. Dimakis and F. Müller-Hoissen, "A noncommutative version of the nonlinear Schroedinger equation," hep-th/0007015; Czech. J. Phys. 51 (2001) 1285.

[8] A. Dimakis and F. Müller-Hoissen, Phys. Lett. A 278 (2000) 139 hep-th/0007074.

[9] A. Dimakis and F. Müller-Hoissen, J. Phys. A 34 (2001) 2571 nlin.si/0008016.

[10] A. Kapustin, A. Kuznetsov and D. Orlov, Commun. Math. Phys. 221 (2001) 385 hep-th/0002193.

[11] K. Takasaki, J. Geom. Phys. 37 (2001) 291 hep-th/0005194.

[12] D. J. Gross and N. A. Nekrasov, JHEP 0007 (2000) 034 hep-th/0005204.

[13] N. A. Nekrasov, "Noncommutative instantons revisited," hep-th/0010017.

[14] M. Legare, "Noncommutative generalized NS and super matrix KdV systems from a noncommutative version of (anti-)self-dual Yang-Mills equations," hep-th/0012077; J. Phys. A 35 (2002) 5489. 
[15] O. Lechtenfeld, A. D. Popov and B. Spendig, JHEP 0106 (2001) 011 hep-th/0103196.

[16] L. D. Paniak, "Exact noncommutative KP and KdV multi-solitons," hep-th/0105185.

[17] O. Lechtenfeld and A. D. Popov, JHEP 0111 (2001) 040 hep-th/0106213; Phys. Lett. B 523 (2001) 178 hep-th/0108118.

[18] K. C. Hannabuss, Lett. Math. Phys. 58 (2001) 153 hep-th/0108228.

[19] O. Lechtenfeld and A. D. Popov, JHEP 0203 (2002) 040 hep-th/0109209.

[20] M. T. Grisaru and S. Penati, Nucl. Phys. B 655 (2003) 250 hep-th/0112246.

[21] S. Bieling, J. Phys. A 35 (2002) 6281 hep-th/0203269.

[22] M. Wolf, JHEP 0206 (2002) 055 hep-th/0204185.

[23] K. Toda, "Extensions of soliton equations to non-commutative $(2+1)$ dimensions," Proceedings of workshop on integrable theories, solitons and duality, Sao Paulo, Brazil, 1-6 July 2002 [JHEP PRHEP-unesp2002/038].

[24] F. Franco-Sollova and T. Ivanova, J. Phys. A 36 (2003) 4207 hep-th/0209153.

[25] Z. Horváth, O. Lechtenfeld and M. Wolf, JHEP 0212 (2002) 060 hep-th/0211041.

[26] K. Furuta, T. Inami and M. Yamamoto, "Topics in nonlinear sigma models in D = 3," hep-th/0211129.

[27] M. Hamanaka and K. Toda, Phys. Lett. A 316 (2003) 77 hep-th/0211148.

[28] I. Cabrera-Carnero and M. Moriconi, "Noncommutative integrable field theories in 2d," hep-th/0211193.

[29] M. Ihl and S. Uhlmann, Int. J. Mod. Phys. B 18 (2003) 4889 hep-th/0211263.

[30] M. Hamanaka and K. Toda, J. Phys. A 36 (2003) 11981 hep-th/0301213.

[31] L. Martina and O. K. Pashaev, "Burgers' equation in non-commutative space-time," hep-th/0302055. 
[32] O. Lechtenfeld and A. D. Popov, "Noncommutative monopoles and Riemann-Hilbert problems," hep-th/0306263.

[33] H. Nishino and S. Rajpoot, Phys. Lett. B 572 (2003) 91 hep-th/0306290.

[34] N. Wang and M. Wadati, J. Phys. Soc. Jap. 72 (2003) 1366; J. Phys. Soc. Jap. 72 (2003) 1881; J. Phys. Soc. Jap. 72 (2003) 3055; J. Phys. Soc. Jap. 73 (2004) 1689.

[35] A. Zuevsky, "Continual Lie algebras and noncommutative counterparts of exactly solvable models," J. Phys. A 37 (2004) 537.

[36] M. Hamanaka and K. Toda, "Towards noncommutative integrable equations," hep-th/0309265.

[37] M. T. Grisaru, L. Mazzanti, S. Penati and L. Tamassia, "Some properties of the integrable noncommutative sine-Gordon system," hep-th/0310214.

[38] R. S. Ward, Phil. Trans. Roy. Soc. Lond. A 315 (1985) 451; "Multidimensional integrable systems," Lect. Notes Phys. 280 (Springer, 1986) 106 "Integrable systems in twistor theory," in Twistors in Mathematics and Physics (Cambridge UP, 1990) 246 [ISBN/0-521-39783-9].

[39] M. Sato, RIMS Kokyuroku 439 (1981) 30; M. Sato and Y. Sato, "Soliton equations as dynamical systems on infinite dimensional Grassmann manifold," in Nonlinear Partial Differential Equations in Applied Sciences (North-Holland, 1983) 259 [ISBN/0-444-86681-7].

[40] B. B. Kadomtsev and V. I. Petviashvili, Sov. Phys. Doklady 15 (1970) 539.

[41] J. Boussinesq, Comptes Rendus Acad. Sci. Paris 72 (1871) 755.

[42] J. Satsuma and R. Hirota, J. Phys. Soc. Jap. 51 (1982) 3390.

[43] K. Sawada and T. Kotera, Prog. Theor. Phys. 51 (1974) 1355.

[44] J. E. Moyal, Proc. Cambridge Phil. Soc. 45 (1949) 99; H. J. Groenewold, Physica 12 (1946) 405.

[45] I. Strachan, J. Geom. Phys. 21 (1997) 255. hep-th/9604142. 
[46] B. Kupershmidt, KP or mKP : noncommutative mathematics of Lagrangian, Hamiltonian, and integrable systems (AMS, 2000) [ISBN/0821814001].

[47] T. Miwa, M. Jimbo and E. Date, (translated by M. Reid), Solitons : differential equations, symmetries and infinite dimensional algebras (Cambridge UP, 2000) [ISBN/0521561612].

[48] M. Błaszak, Multi-Hamiltonian Theory of Dynamical Systems (Springer, 1998) [ISBN/3-540-64251-X].

[49] Y. Ohta, J. Satsuma, D. Takahashi and T. Tokihiro, Prog. Theor. Phys. Suppl. 94 (1988) 210.

[50] I. M. Gelfand and L. A. Dikii, Funct. Anal. Appl. 10 (1976) 13 (Russian), 259 (English).

[51] B. A. Kupershmidt, Commun. Math. Phys. 99 (1988) 51.

[52] J. Matsukidaira, J. Satsuma and W. Strampp, J. Math. Phys. 31 (1990) 1426.

[53] G. Segal, "Integrable systems and inverse scattering," in Integrable Systems (Oxford UP, 1999) 53 [ISBN/0-19-850421-7].

[54] M. Jimbo and T. Miwa, Publ. Res. Inst. Math. Sci. Kyoto 19 (1983) 943.

[55] M. J. Ablowitz, D. J. Kaup, A. C. Newell and H. Segur, Stud. Appl. Math. 53 (1974) 249.

[56] F. Calogero, Lett. Nuovo Cim. 14 (1975) 443; O. I. Bogoyavlenskii, Math. USSR-Izv. 34 (1990) 245; J. Schiff, "Integrability of Chern-Simons-Higgs vortex equations and a reduction of the selfdual Yang-Mills equations to three-dimensions," NATO ASI Ser. B 278 (Plenum, 1992) 393.

[57] G. Wilson, Math. Proc. Cambridge Phil. Soc. 86 (1979) 131.

[58] G. Wilson, Ergod. Th. \& Dynam. Sys. 1 (1981) 361.

[59] V. G. Drinfeld and V. V. Sokolov, J. Sov. Math. 30 (1984) 1975. 
[60] R. Hirota, Phys. Rev. Lett. 27 (1971) 1192.

[61] E. Date, M. Jimbo, M. Kashiwara and T. Miwa, "Transformation groups for soliton equations," in Nonlinear integrable systems - classical theory and quantum theory (World Sci., 1983) 39 [ISBN/9971950324] and references therein.

[62] T. Ikeda and K. Takasaki, Int. Math. Res. Notices 7 (2001) 329 nlin.si/0004015; S. Kakei, T. Ikeda and K. Takasaki, Annales Henri Poincare 3 (2002) 817 nlin.si/0107065.

[63] A. Dimakis and F. Muller-Hoissen, J. Phys. A 37 (2004) 4069 hep-th/0401142; J. Phys. A 37 (2004) 10899 hep-th/0406112; "Extension of Moyal-deformed hierarchies of soliton equations," nlin.si/0408023.

[64] N. Wang and M. Wadati, J. Phys. Soc. Jap. 73 (2004) 1689.

[65] M. Sakakibara, J. Phys. A 37 (2004) L599 nlin.si/0408002.

[66] L. D. Faddeev and L. A. Takhtajan, Hamiltonian Methods in the Theory of Solitons (Springer, 1987) [ISBN/0-387-15579-1].

[67] M. J. Ablowitz and P. A. Clarkson, Solitons, Nonlinear Evolution Equations and Inverse Scattering (Cambridge UP, 1991) [ISBN/0-521-38730-2]; L. J. Mason and N. M. Woodhouse, Integrability, Self-Duality, and Twistor Theory (Oxford UP, 1996) [ISBN/0-19-853498-1]; M. J. Ablowitz, S. Chakravarty and L. A. Takhtajan, Commun. Math. Phys. 158 (1993) 289; T. A. Ivanova and A. D. Popov, Theor. Math. Phys. 102 (1995) 280 [Teor. Mat. Fiz. 102 (1995) 384]; M. J. Ablowitz, S. Chakravarty and R. G. Halburd, J. Math. Phys. 44 (2003) 3147.

[68] O. Lechtenfeld, A. D. Popov and B. Spendig, Phys. Lett. B 507 (2001) 317 hep-th/0012200.

[69] H. Ooguri and C. Vafa, Mod. Phys. Lett. A 5 (1990) 1389; Nucl. Phys. B 361 (1991) 469; Nucl. Phys. B 367 (1991) 83. 